\title{
A FRAMEWORK FOR SEMIQUALITATIVE REASONING IN ENGINEERING APPLICATIONS
}

\author{
RAFAEL M. GASCA, J.A. ORTEGA, and M.TORO Dpto. Lenguajes y Sistemas \\ Informáticos, Facultad de Informática y Estadística, University of Seville, Spain
}

In most cases the models for experimentation, analysis, or design in engineering applications take into account only quantitative knowledge. Sometimes there is a qualitative knowledge that is convenient to consider in order to obtain better conclusions. These qualitative concepts can be labels such as "high," "very negative," "little acid," "monotonically increasing"' or

symbols such as $\gg, \approx$, etc... Engineers have already used this type of knowledge implicitly in many activities. The framework that we present here lets us express explicitly this knowledge.

This work makes the following contributions. First, we identify the most important classes of qualitative concepts in engineering activities. Second, we present a novel methodology to integrate both qualitative and quantitative knowledge. Third, we obtain signifi cant conclusions automatically. It is named semiqualitative reasoning.

Qualitative concepts are represented by means of closed real intervals. This approximation is accepted in the area of Artifi cial Intelligence. A modeling language is specifi ed to represent qualitative and quantitative knowledge of the model. A numeric constraint satisfaction problem is obtained by means of corresponding rules of transformation of the semantics of this language. In order to obtain conclusions, we have developed algorithms that treat the problem in a symbolic and numeric way. The interval conclusions obtained are transformed into qualitative labels through a linguistic interpretation. Finally, the capabilities of this methodology are illustrated on different problems.

A model is the representation of the relevant aspects of a determined system constructed for the purpose of answering particular questions. In many systems, the lack of quantitative information and sometimes, also, the "excess" of quantitative information is frequent. There is the confirmation that in certain situations it is possible to obtain significant conclusions from a purely qualitative knowledge. Qualitative reasoning $(Q R)$ is a well-defined

Address correspondence to Rafael M. Gasca, Dpto. Lenguajes y Sistemas Informáticos, Facultad de Informática y Estadística, University of Seville, Avda Reina Mercedes s/n 41012 Seville, Spain. E-mail: gasca, ortega, mtoro@1si.us.es 
method for dealing with these qualitative models. In the eighties, the main concepts of the qualitative techniques appeared in the area of Artificial Intelligence $(A I)$, by means of the publication of the systems ENVISION (DeKleer and Brown 1984), QSIM (Kuipers 1986) and QPT (Forbus 1984). In the last decade, different monographs have been published (Kuipers 1994; Piera 1995; Dague 1995; Travé-Massuyes, Dague, and Guerrin 1997; Ortega 2000) that tackle the topics and the different developed techniques in qualitative reasoning. This qualitative knowledge is useful to understand, in a simple way, some of the properties of the models, therefore the simplicity is its principal property. The qualitative representation of the world and the attempt to qualitatively reason about the world are not new and have been used in different engineering tasks, such as diagnosis (Biswas, Kapadia, and Yu 1999), supervision (Bousson 1993; Moreno 1993), control (Foulloy 1993), conceptual design (Bozzo et al. 1998), analysis (Aracil and Toro 1993; Gasca 1998; Lee 2000), fault detection (Armengol 2000) and temporal behavior patterns (Ortega 2000). In most of the approaches to modeling qualitative reasoning in $A I$, it is explicitly or implicitly assumed that qualitative models are obtained directly from quantitative models, or at least could be refined to a quantitative description of such a system. Due to this, it may seem that $Q R$ would compete with different fields and scientific methods. The question is then how the results obtained by $Q R$ relate to those derived from the corresponding quantitative analysis.

In the formalization of $Q R$ (Struss 1989), the decisions about how to describe the qualitative quantities and to manipulate them to obtain results are:

- A finite set of values.

- The set of qualitative values must cover the whole range of the interesting behaviors.

- An interpretation of the results obtained by the qualitative analysis.

- A natural order of the qualitative values.

- A formalism that expresses the description of the system in terms of relationships between qualitative variables and operations to obtain the solutions.

Different approaches have been proposed to apply qualitative reasoning to problems of engineering. They had important difficulties, since a lot of importance is given to simple parameters and the relationships among them are generally rejected. In these problems, apart from signs of quantities, it also seems convenient to consider the absolute or relative order of magnitude of the quantities. For example, if $A$ is big and $B$ is big, the sum is easy to infer that its result is big but if the subtraction is carried out, the result may be big, medium, or small. This fact together with other 
considerations has given rise to new approximations to formalize structures and tools for $Q R$.

The order of magnitude formalisms have been introduced as alternatives to pure qualitative theories in order to address the problems deriving from the poor expressivity of qualitative theories, leading to what is known as the over-abstraction phenomenon (Kuipers 1986). The absolute order of magnitude is based on the sign algebra, where quantities are given by signs. Other important algebras are qualitative order of magnitude algebras (Travé-Massuyes and Piera 1989; Missier, Piera, and Trave 1989; Piera, Sanchez, and Travé-Massuyes 1991; Agell 1998) and hybrid sign-real algebra (Williams 1991), that explains how it is possible to do certain algebraic treatments on constraints in the real algebra, and then map the constraints into the sign domain for other inferences.

Another approach that offers a midway abstraction level between numerical methods and qualitative formalisms is the relative order of magnitude reasoning. The qualitative knowledge is represented by binary relations expressing orders of magnitude between two quantities (e.g. 'close to,' 'negligible,' 'distant of'). The first attempt to formalize such reasoning appeared with the formal system FOG (Raiman 1986) based on three basic relations and 32 rules. Nevertheless, limitations of FOG have been pointed out in the formalism $O(M)$ (Mavrovouniotis and Stephanopoulos 1990), which prevents it from its real use in engineering. $O(M)$ considers seven binary primitive relations between positive quantities, with interval semantics. Inference strategies are based on propagation of order of magnitude relations through properties of the relations, solved or unsolved algebraic constraints. In $O(M)$ there is the impossibility, at a formal level, to express a gradual change from one order of magnitude to another, due to the non-overlapping nature of the orders of magnitude. Then a new formal system $R O M(K)$ (Dague 1993) is proposed to introduce a new relation 'distant from'. Determined qualitative labels of $\operatorname{ROM}(K)$ correspond to the sets Small and Rough previously defined in a set-based general framework (Raiman 1991), that uses a coarse equilibrium which weighs quantities with a variable level of precision.

In previous techniques, the two most important problems are the difficulty to incorporate quantitative knowledge, when available, and the difficulty to control the inference process, in order to obtain valid results in the real problems. The measure of accuracy of the inferences can solve some problems. It was proposed first for FOG (Dubois and Prade 1989) and more recently for $R O M(K)$ (Dague 1993). These extensions may cause a spurious result produced in the reasoning process. In order to avoid such a problem, a refined definition of the negligibility relation was proposed (Dollinger and Letia 1998). Also, the chance of describing, in a qualitative way, the natural 
grading of negligibility obtains a greater precision in particular problems (Sánchez, Prats, and Piera 1996b).

The first works about the integration of the qualitative and quantitative knowledge are $O(M)$ and $R O M(\mathbb{R})$ (Dague 1993), but the obtained results, although sound, are not in general optimal. Later, another reasoning method proposed to integrate absolute and relative order of magnitude relations in qualitative models (Sánchez, Prats, and Piera 1996a). The last works are aiming at formalizing reasoning when we dispose of some real quantitative data and only dispose of the qualitative descriptions of other ones. Numerical and qualitative techniques are combined to make more significant inferences in static systems (Gasca, Toro, and Ortega 1996; Sanchez, Prats, and Piera 1998; Gasca 1998) and in systems that change over time (Berleant and Kuipers 1997; Gasca 1998; Armengol 2000; Ortega 2000). In this work we will only study the first systems.

Reasoning on the ranges of values of variables is another type of reasoning often used in qualitative systems, where there are inaccurate data or partially defined parameters. It can be generalized to a Numeric Constraint Satisfaction Problem $(N C S P)$. It is a triple $(X, D, C)$ where $X$ denotes a set of variables, $D$ denotes a set of domains containing all acceptable values for every variable and $C$ is the conjunction of constraints that have to be satisfied. A natural way of reasoning on the ranges of values is to propagate the domains of the variables through the constraints. Then consistency techniques have been applied to NCSP to detect inconsistent values and delete them. Different techniques have been proposed in the bibliography (Davis 1987; Hyvönen 1992; Lhomme 1993, 1994; Van Hentenryck, McAllester, and Kapur 1995; Benhamou and Granvillers 1996; Van Hentenryck, Michel, and Deville 1997; Marti and Rueher 1997; Jussien and Lhomme 1998). A lot of these techniques have a major drawback, since they introduce choice points. The efficiency of some previous algorithms is analyzed in a recent work (Collavizza, Delobel, and Rueher 1999).

In this work, our main aim is reasoning in static models, where some real quantitative data and qualitative descriptions of other ones are known (semiqualitative models). We integrate several reasoning techniques. The main motivation for integrating several reasoning techniques is to achieve a synergy which produces results that could not be obtained if each mode were operating individually. It is possible by means of the construction of a novel reasoning framework, where we can easily express these models in a declarative way, what provides a substantial expressive power. Inference strategies within this framework are separated into two steps: symbolic and numerical. They constrain the inferences efficiently and obtain results which are suitable for many engineering activities. 


\section{SEMIQUALITATIVE REASONING}

The representation of qualitative concepts can be selected within different options. Nevertheless a widely accepted representation of these concepts may be closed real intervals. For example the colors green, yellow, and red can be associated to their respective interval wavelengths. However, this representation has difficulties such as to express the gradual changes of a value towards one another. The approximation of interpreting the qualitative predicates as an interval may become practical and useful, but different problems in interval-based reasoning (Struss 1990) were identified.

It is important to establish a mapping between the qualitative descriptions and numerical intervals. This topic has been shortly treated in the bibliography. In this work the assignment of intervals is suppose to be made considering the experience of the experts. In complex systems, every interval must be compatible with the rest of the intervals that are being defined in the model. It is usually a difficult task, as different experts participate in the modeling, then iterative processes are required until the intervals are assigned satisfactorily for all the qualitative labels. Once their representation for the different variables and relationships is chosen, the equations of the models can be reduced to a set of constraints between the qualitative and quantitative variables and parameters.

Our reasoning framework obtains conclusions in the previous semiqualitative models. Firstly, it is convenient to consider the type of representation of qualitative knowledge. These concepts are represented, in this work, as closed real intervals and their computational treatment can be accomplished by means of numerical methods. Secondly, we have developed ad hoc algebraic and numerical methods to improve the efficiency and precision of the results. The chosen representation for the qualitative characteristics of a model allows us to define a specification language. The users of the system can easily introduce qualitative and quantitative description and what they would like to know of the analyzed system.

The implementation of the reasoning procedures is inspired from wellknown works in AI about Constraint Satisfaction Problem. In Figure 1, we show that the selection of a semiqualitative model is carried out starting from

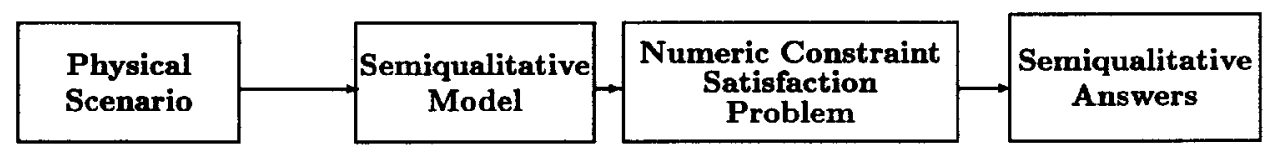

FIGURE 1. Diagram of the semiqualitative reasoning. 
a certain physical scenario. These models have their foundation in real systems. A group of experts should inform of the set of constraints and qualitative values that represent the reality. A semiqualitative model defines a space of models that includes a set of different precise models of the real system. These models are generally constituted by a constraint network. Qualitative elements are transformed into intervals and semiqualitative reasoning obtains a space of solutions for the queries.

This investigation begins mainly motivated by the lack of data to build conventional quantitative models, or because we want to absorb a certain type of qualitative knowledge from the quantitative knowledge. For example, the acidity of a solution can be predicted in the analysis of chemical systems, finding the concentration of $\mathrm{H}^{+}$ions. When the equilibrium of simple acid dissolved in water is reached, then the concentration of the different species is governed by the following set of four equations:

Charge balance: $\left[\mathrm{H}^{+}\right]=\left[\mathrm{A}^{-}\right]+\left[\mathrm{OH}^{-}\right]$

Mass Balance: $c=\left[A^{-}\right]+[A H]$

Acid Equilibrium: $K_{a}[A H]=\left[A^{-}\right]\left[H^{+}\right]$

Water Equilibrium: $10^{-14}=\left[O H^{-}\right]\left[H^{+}\right]$

where $c$ is the initial concentration of the acid, $K_{a}$ is an equilibrium constant and square brackets stand for the concentration of the species.

For example, in this model we may know the qualitative value of $c$ as "diluted" and the qualitative value of $K_{a}$ as "weak." Semiqualitative reasoning consists of the application of a set of techniques to solve queries of engineers, studying the qualitative and/or quantitative values that satisfy the specified constraints. The queries may be:

- Boolean Query: Its answer is TRUE or FALSE. The result is true if the constraint network that represents the semiqualitative model is satisfied. For the previous example a boolean query may be: Is the qualitative value of $\left[\mathrm{H}^{+}\right]$similar to the qualitative value of $\left[A^{-}\right]$?. The boolean operators and and or may be also used in these queries.

- Qualitative Query: The answer is the qualitative label of a variable, relation o boolean composition of relations, such that the range of values of the answer satisfies all the constraints. For the previous example a qualitative query may be: What is the qualitative value of $\left[H^{+}\right]$?

This framework for semiqualitative reasoning lets us obtain quantitative answers as precise real intervals. An appropriate interpretation of these answers is necessary to obtain the corresponding qualitative label. Next, we 
propose a language to express qualitative and quantitative knowledge of a model and a set of symbolic and numerical techniques to obtain the answers to the previous queries.

\section{SPECIFICATION OF THE SEMIQUALITATIVE MODELING LANGUAGE}

Modeling language must describe the identified qualitative knowledge of the models. Also, it must provide a declarative semantics for the solving process. First, we will describe the abstract syntax of the language and later its semantics.

\section{Syntax}

The used notation of the language is illustrated in the Figure 2 by means of the corresponding syntactic categories. These syntactic categories, possibly subscript and/or superscript, stand for instances of them or components of them. The syntax of the language is defined by means of the abstract syntax illustrated in Figure 3. In this grammar the operator or is represented by means of ';' and the operator and by means of ','. In this work, they are both represented in the two ways without any distinction.

\section{Syntactic Categories}

- Arithmetic Operators represent a set of unary and binary arithmetic operators. An example of these operators may be: $B_{a}=\{+,-, *, /, \ldots\}$.

- Qualitative Operators, $U$ and $B$, represent a set of unary and binary operators for every qualitative magnitude of the problem, respectively. For example $U_{q}=\{$ large, small, medium, negative, short, acid, high, ... $\}$ and $\quad B_{q}=\{$ much smaller than than, moderately smaller than, slightly smaller than, exactly equal to, much larger than, negligible, distant from $\ldots\}$.

\begin{tabular}{llll}
\hline $\mathbf{x}$ & variables & $\mathrm{e}$ & expressions \\
$\mathbf{v}$ & real values & $\mathrm{f}$ & functions \\
$\mathrm{I}$ & interval values & $\mathrm{g}$ & envelope functions \\
$u_{a}$ & arithmetic unary operators & $\mathrm{p}$ & predicates \\
$b_{a}$ & arithmetic binary operators & $\mathrm{c}$ & constraints \\
$u_{q}$ & qualitative unary operators & $\mathrm{q}$ & single queries \\
$\boldsymbol{b}_{q}$ & qualitative binary operators & $\mathrm{cq}$ & compound queries \\
\hline
\end{tabular}

FIGURE 2. Syntactic categories. 


\begin{tabular}{|c|c|c|c|c|c|c|c|c|}
\hline \multirow[t]{8}{*}{ e } & : & $\mathrm{v}$ & $\mathrm{p}$ & : & $\overline{u_{q}(e)}$ & $q$ & : & $\overline{e ?}$ \\
\hline & 1 & I & & 1 & $b_{q}(e, e)$ & & 1 & $e ? e$ \\
\hline & 1 & $\mathbf{x}$ & & ; & & & 1 & $?$ \\
\hline & 1 & $u_{a}(e)$ & c & : & $p$ & & ; & \\
\hline & 1 & $b_{a}(e, e)$ & & 1 & $c, c$ & cq & : & $q$ \\
\hline & i & $\mathbf{f}(\mathbf{e})$ & & 1 & $c ; c$ & & 1 & $c q, c q$ \\
\hline & 1 & $\mathbf{g}(\mathbf{e})$ & & ; & & & 1 & $c q ; c q$ \\
\hline & ; & & & & & & ; & \\
\hline
\end{tabular}

FIGURE 3. Abstract syntax.

- Functions and Envelope Functions, let $\mathbb{R}$ be the set of real numbers, then $F$ represents a set of continuous functions $f: \mathbb{R} \rightarrow \mathbb{R}$. These functions are described by means of the tuple $f(x) \equiv\left\langle e(x), I_{1}, I_{2}\right\rangle$, where $I_{1}$ and $I_{2}$ are the domain and range of $f$ respectively. An envelope function $g$ represents a family of functions between two functions $f_{1} \in F$ and $f_{2} \in F$,

$$
g(x) \equiv\left\langle f_{1}(x), f_{2}(x), I_{1}, I_{2}\right\rangle \quad \text { so that } \quad \forall x \in I_{1}: f_{1}(x)<f_{2}(x)
$$

where $I_{1}$ and $I_{2}$ stand for the domain and range of $g$ respectively.

- Predicates, represented by $P$, where each $p_{i}$ can be a unary predicate $u_{i}(e)$, where $u_{i} \in U$, that represents the qualitative knowledge of the expression $e$, or a binary predicate $b_{i}\left(e_{1}, e_{2}\right)$, where $b_{i} \in \mathcal{B}$, that represents the qualitative relationship between the values of $e_{1} \mathrm{y} e_{2}$.

- Constraints, represented by $C$, are predicates, or boolean combination of predicates that satisfy all the values of the model.

- Single Queries, $Q$ where every $q_{i}$ is a query. It may be a unary query, such as $e$, that indicates the qualitative value of the expression $e$ or a binary query such as $e_{1} ? e_{2}$, which indicates the relative order of magnitude relation between $e_{1}$ and $e_{2}$. The query ? represents a question about the consistency of the model.

- Compound Queries are boolean expressions of queries.

\section{Semantics}

The semantics of the language is defined by means of a set of rules of transformation. They transform an initial model into a normalized one. If $r$ always denotes a new variable and $I$ represents a real interval, the transformations applied to the initial model are the following: 
- Rename constants of the model that are intervals: Every constant of the model is substituted by a variable and a constraint.

$$
C(\ldots, I, \ldots) \equiv C(\ldots, r, \ldots), \quad r \in I
$$

- Semantics of unary predicates: The following transformation is carried out

$$
u_{q}(e) \equiv e-r=0, \quad r \in I_{u}
$$

where $I_{u}$ is the corresponding interval to the unary operator $u \in U$. This transformation is carried out to express the qualitative knowledge that somebody has about the expression. In the bibliography there are different spaces of qualitative description. One of them uses two landmarks, denoted as $\alpha$ and $\beta$ (Travé-Massuye's and Piera 1989) and the other uses more landmarks (Agell 1998). It depends on every magnitude of the reasoning problem and the level of precision to denote a quantity. This association between operators and intervals is carried out according to the knowledge of the expert. The absolute order of magnitude scale for every quantity of the model must be coherent with the corresponding relative order of magnitude scale.

- Semantics of binary predicates: These predicates are related to the division and they have the following semantics

$$
b_{q}\left(e_{1}, e_{2}\right) \equiv e_{1}-e_{2} * r=0, \quad r \in I_{b}
$$

$I_{b}$ is the interval corresponding to symbol $b$. In the bibliography there are different spaces of relative order of magnitude description. One of them uses one tolerance parameter (Mavrovouniotis and Stephanopoulos 1990) and the other uses two parameters (Dague 1993). This may express a gradual change from one order of magnitude to another and the first one may not express it.

- Semantics of functions and envelope functions: According to the definition of these functions, the following transformation is applied

$$
\begin{aligned}
& r=f(x) \equiv\left\{\begin{array}{l}
f(x)-r=0 \\
x \in I_{1}, r \in I_{2}
\end{array}\right. \\
& r=g(x) \equiv\left\{\begin{array}{l}
g(x)-r=0 \\
g(x)=r_{1} g(x)+\left(1-r_{1}\right) * \bar{g}(x) \\
r_{1} \in[0,1], x \in I_{1}, r \in I_{2}
\end{array}\right.
\end{aligned}
$$


The envelope functions express qualitative aspects, and represent a family of functions enveloped in an upper function $\bar{g}: \mathbb{R} \rightarrow \mathbb{R}$ and lower one $\underline{g}: \mathbb{R} \rightarrow \mathbb{R}$. The family of functions is defined by the expression:

$$
g(x)=\alpha \underline{g}(x)+(1-\alpha) \bar{g}(x), \quad \alpha \in[0,1], \quad x \in I_{1}
$$

Then, if $\alpha=0 \Rightarrow g(x)=\bar{g}(x)$ and if $\alpha=1 \Rightarrow g(x)=g(x)$ and any other value of $\alpha$ belonging to the interval [0,1] represents a set of values between $\underline{g}(x)$ and $\bar{g}(x)$.

- Semantics of single query: Depending on the type of query, the following transformation is carried out

$$
e_{1} ? \equiv\left\{\begin{array} { l } 
{ e _ { 1 } - r = 0 } \\
{ r ? }
\end{array} \quad e _ { 1 } ? e _ { 2 } \equiv \left\{\begin{array}{l}
e_{1}-e_{2} * r=0 \\
r ?
\end{array}\right.\right.
$$

- Semantics of compound query: For all the single queries the previous transformation is carried out and the boolean operators are not transformed.

\section{Semiqualitative Models}

Our language lets us specify the model of the system with qualitative and quantitative knowledge by means of the following constraint diagram

$$
M_{0} \equiv\left\{\begin{array}{l}
C=\left\{c_{1}, \ldots, c_{t}\right\} \\
V=\left\{x_{1}, \ldots, x_{n}\right\} \\
Q=\left\{q_{1}, \ldots, q_{m}, c q_{1}, \ldots, c q_{p}\right\}
\end{array}\right.
$$

where $C$ is the set of constraints of the model, $V$ is the set of variables of the constraints, and $Q$ is the set of queries, represented according to the syntax of the language. Therefore semiqualitative reasoning is considered as the methodology to obtain values of $Q$ so that all the constraints belonging to $C$ are satisfied.

In Figure 4 is shown a countercurrent heat-exchanger. It is commonly studied in order of magnitude reasoning (Mavrovouniotis and Stephanopoulos 1990; Dague 1993b; Sánchez, Prats, and Piera 1998). The important variables in the analysis of the device are the molar-heat $\mathrm{KH}$ and the molar-fowrate $\mathrm{FH}$ of the hot stream, and the molar-heat $K C$ and the molar-flowrate $F C$ of the cold stream. Also, the temperature differences have been named $D T H=T h 1-T h 2$, $D T C=T c 1-T c 2, \quad D T 1=T h 1-T c 1, \quad D T 2=T h 2-T c 2 . \quad D T H$ is the temperature drop of the hot stream, $D T C$ is the temperature rise of the cold stream, $D T 1$ and $D T 2$ are the driving force at the left and right end of the device 


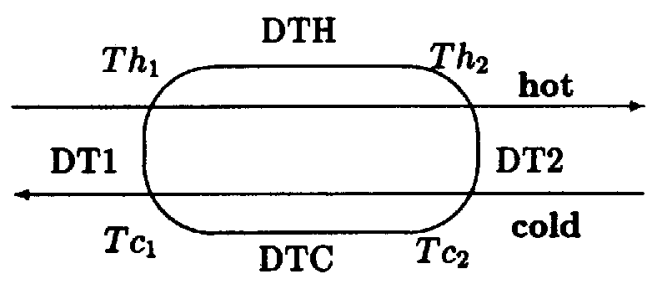

FIGURE 4. A countercurrent heat-exchanger.

respectively. In order to make the example clearer, the definition of these temperature differences is chosen so that they are positive. Some constraints of the problem are:

$$
\begin{aligned}
& D T H K H F H=D T C K C F C \\
& D T H-D T 1-D T C+D T 2=0
\end{aligned}
$$

The first equation is the energy balance of the device and the second equation is the result of the definition of the temperature differences. In a particular case, the following qualitative relations in order of magnitude and quantitative relations may be known: DT2 is moderately smaller than DT1, DT1 is much smaller than DTH and $K H$ is 1.1 times larger than $K C$. The query is the obtaining of the qualitative relation between $F C$ and $F H$ in this model. The specification of this problem in the proposed language is:

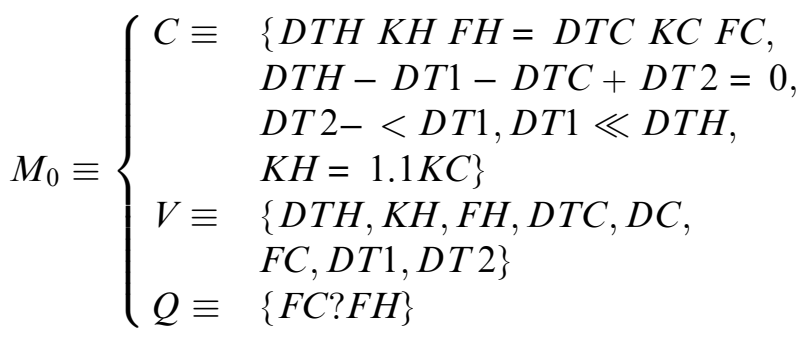

In order to improve the computational treatment of the problem, this initial model $M_{0}$ is transformed into a new model $M_{1}$, where the set of variables $V$ is divided into three sets:

- $Y$, which represents the set of variables of a well-known range.

- $X$, which represents the set of variables whose domains we would like to know and that satisfy all the constraints.

- $Z$, which represents the rest of the variables whose range we do not know, nor we are interested in. 
According to it and the transformation rules of the semantics of the language, the model is transformed into the new model $M_{1}$

$$
M_{1} \equiv\left\{\begin{aligned}
C=\quad & \text { DTC FC KC+DTH FH KH=0, } \\
& -D T C+D T H-D T 1+D T 2=0, \\
& D T 2-D T 1 r_{1}=0, D T 1-D T H r_{2}=0 \\
& K H-1.1 K C=0, F C-F H r_{3}=0 \\
& \left.r_{1} \in I_{-<}, r_{2} \in I_{\ll}\right\} \\
V=\{Z=\{D T H, K H, F H, D T C, D C, F C, D T 1, D T 2\} & \left.Y=\left\{r_{1}, r_{2}\right\}, X=\left\{r_{3}\right\}\right\} \\
Q= & r_{3} ?
\end{aligned}\right.
$$

This model $M_{1}$ may be considered as a numeric constraint satisfaction problem that integrates qualitative and quantitative knowledge. The reasoning process must use an adequate methodology in order to obtain significant conclusions.

\section{SEMIQUALITATIVE REASONING FRAMEWORK}

A fundamental aspect of this reasoning framework is the inference methodology. We propose a methodology that uses constraint networks. In $A I$, it is known as constraint-based reasoning (Freuder and Mackworth 1994). The proposed modeling language generates a numeric constraint satisfaction problem. The solving of this NCSP lets us answer the user's queries. The answers are qualitative or quantitative, depending on the user's interest. Our methodology obtains the interval value or set of values corresponding to the proposed queries following two well differentiated steps. The general architecture is shown in Figure 5. In the first step, named Symbolic Processing, we use symbolic techniques to transform the initial constraint network $C N$, that represents the model $M_{1}$, into a new constraint network $C N_{1}$. It allows a more efficient resolution of the proposed queries in the following step. The second step is a numerical treatment of $C N_{1}$, where we use techniques of interval reasoning until obtaining the solutions Sol.

In the Symbolic Processing step, we have implemented the following algorithms of symbolic transformation: algorithms of elimination of constraints and substitution of variable, symbolic generation of constraints and

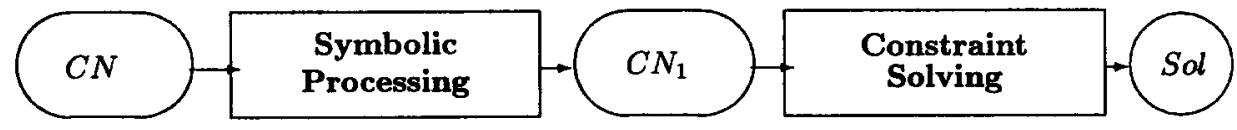

FIGURE 5. Diagram of computational processing for semiqualitative reasoning. 
transformation of factored constraints. In the constraint solving step, we apply branch and prune algorithms with efficient constraint narrowing operators, where it should be considered that the speed of convergence depends, in an important way, on the algebraic ways of constraints (Ratschek and Rokne 1984). The aim of this treatment is the application of the appropriate algorithms to increase the efficiency and to obtain the most precise ranges of values of the queries. The results of the proposed methodology are the set of answers to the user's queries.

\section{Symbolic Processing}

The previous model $M_{1}$ could be treated numerically as a numeric constraint satisfaction problem, but in a lot of models the obtained results, although sound, are not in general optimal. Therefore we propose a previous symbolic processing in order to transform the constraint network into another which has the same solutions as the previous one. This symbolic processing is essentially developed to obtain more precise ranges of the solutions of the model and improve the computational behavior of the numerical algorithms.

\section{Symbolic Reduction}

This algorithm carries out the elimination of determined constraints where there are non-significant variables. These variables are those whose values are neither known nor important in the resolution of the problem. Therefore, this procedure can reduce the number of variables that you have in the model.

The process of symbolic reduction is applied to the constraints of equality of the model. To take the operational aspects of symbolic reduction into account we define the following concepts:

- Definition 1 (Removed Variable) A removed variable is a variable $z \in Z$ which appears in a constraint in the following way $k z+e(x)=0$, where $k$ is a real constant. In such cases the symbolic substitution $s$ is $s \equiv z=-e(x) / k$

- Definition 2 (Removed Constraint) A removed constraint is a constraint with removed variables.

- Definition 3 (Interference of constraint respect to a removed variable) The Interference of a constraint respect to a removed variable is the number of constraints where the removed variable is present, except the considered constraint.

$$
\text { Interference }\left(c_{i}, v_{i}\right)=\mathcal{N} j: j \in\{1, \ldots, n\} \bullet\left(v_{i} \in \operatorname{Var}\left(c_{j}\right) \wedge j \neq i\right)
$$


where $\mathcal{N}$ stands for the counter operator and $\operatorname{Var}$ is the set of variables of a constraint.

- Definition 4 (Interference of a constraint) The Interference of a constraint is the sum of the interferences of all removed variables for the constraint.

$$
\text { Interference }\left(c_{i}\right)=\sum_{v_{j} \in \operatorname{Var}\left(c_{j}\right) \wedge \operatorname{removed}\left(v_{j}\right)} \operatorname{Interference}\left(c_{i}, v_{j}\right)
$$

The following algorithm removes determined constraints and variables:

\title{
begin
}

while there are removed constraints

To choose a removed constraint $c$ according to some criterion

To eliminate $c$ of the constraint network

To choose a removed variable $v$ of the constraint $c$ according to some criterion

To substitute $v$ by the symbolic expression $s$ in the constraint network

\author{
endwhile \\ end
}

The possible criteria of selection of the constraints to be removed are:

- That the constraint may be removed.

- To choose the constraint that has the minimum interference.

- If the interference is the same for two or more constraints then the firstly written constraint will be removed.

The possible criteria of selection of variable to be removed are:

- To choose the variable with less interference and different of zero in the first place.

- To use the lexicographic sort when two or more variable have the same interference.

\section{Application to countercurrent heat-exchanger}

The previous model $M_{1}$ was formulated in the section of the specification language. The variables of the model have been renamed to clarify the diagram. Initially, the domain of the question $r_{3}$ is defined as $[-\infty,+\infty]$. The model $M_{1}$ is then: 


$$
M_{1} \equiv\left\{\begin{aligned}
C \equiv\left\{\begin{array}{l}
x_{8}-x_{7} r_{1}=0, x_{7}-x_{1} r_{2}=0 \\
x_{2}-1.1 x_{5}=0, x_{6}-x_{3} r_{3}=0 \\
-x_{4}+x_{1}-x_{7}+x_{8}=0,-x_{4} x_{6} x_{5}+x_{1} x_{3} x_{2}=0
\end{array}\right. \\
V \equiv\left\{\begin{array}{l}
Z=\left\{x_{1}, x_{2}, x_{3}, x_{4}, x_{5}, x_{6}, x_{7}, x_{8}\right\} \\
Y=\left\{r_{1}, r_{2}\right\} \\
X=\left\{r_{3}\right\}
\end{array}\right. \\
D \equiv\left\{r_{1} \in I_{-<}, r_{2} \in I_{\ll}, r_{3} \in[-\infty,+\infty]\right.
\end{aligned}\right.
$$

In the first iteration of the previous algorithm, the removed variables and interference of the constraints are obtained. The results are shown in Table 1. According to previous criteria of selection, the first written constraint is removed. Table 2 shows the new constraint network and the new interference of the removed variables and constraints. In the last iteration, a new constraint network with an only constraint is obtained.

$$
1.1 x_{1} x_{3} x_{5}-r_{3}\left(x_{1}-r_{2} x_{1}+r_{1} r_{2} x_{1}\right) x_{3} x_{5}=0
$$

and the symbolic simplification is $x_{1}\left(1.1-r_{3}+r_{2} r_{3}-r_{1} r_{2} r_{3}\right) x_{3} x_{5}=0$. The new model $M_{2}$ is then

$$
M_{2} \equiv\left\{\begin{array}{l}
C \equiv\left\{\left(1.1-1 r_{3}+r_{2} r_{3}-r_{1} r_{2} r_{3}\right) x_{1} x_{3} x_{5}=0\right\} \\
V \equiv\left\{r_{1}, r_{2}, r_{3}\right\} \\
D \equiv\left\{r_{1} \in I_{-<}, r_{2} \in I_{\ll}, r_{3}, x_{1}, x_{3}, x_{5} \in[-\infty, \infty]\right\}
\end{array}\right.
$$

The initial $C N$ has been reduced to a new constraint network with an only constraint that contains the variables $r_{1}, r_{2}$ and the variable $r_{3}$ that represents the query. Most of the non-significant variables of the model have been eliminated and therefore it will improve the obtaining of globally consistent solutions.

In a general case, the application of symbolic reduction will obtain several constraints. The application of the same symbolic reduction to the problem of equilibrium of a weak acid obtains the following results:

$$
\begin{gathered}
K_{a}\left(c-\left[\mathrm{H}^{+}\right]+\left[O H^{-}\right]\right)-\left(\left[H^{+}\right]-\left[O H^{-}\right]\right)\left[H^{+}\right]=0, \\
10^{-14}-\left[O H^{-}\right]\left[H^{+}\right]=0
\end{gathered}
$$

TABLE 1 First Iteration of Symbolic Reduction Applied to the Model of Countercurrent Heat-exchanger

\begin{tabular}{lcc}
\hline$c_{i}$ & Removed Variables/Interference & Interference $\left(\mathrm{c}_{\mathrm{i}}\right)$ \\
\hline$-r_{1} x_{7}+x_{8}=0$ & $\left\{x_{8} / 1\right\}$ & 1 \\
$-r_{2} x_{1}+x_{7}=0$ & $\left\{x_{7} / 2\right\}$ & 2 \\
$x_{2}-1.1 x_{5}=0$ & $\left\{x_{2} / 1, x_{5} / 1\right\}$ & 2 \\
$-r_{3} x_{3}+x_{6}=0$ & $\left\{x_{6} / 1\right\}$ & 1 \\
$x_{1}-x_{4}-x_{7}+x_{8}=0$ & $\left\{x_{1} / 2, x_{4} / 2, x_{7} / 2, x_{8} / 1\right\}$ & 6 \\
$x_{1} x_{2} x_{3}-x_{4} x_{5} x_{6}=0$ & $\emptyset$ & \\
\hline
\end{tabular}


TABLE 2 Second Iteration of Symbolic Reduction Applied to the Model of Countercurrent Heat-exchanger

\begin{tabular}{lcc}
\hline$c_{i}$ & Removed Variables/Interference & Interference $\left(\mathrm{c}_{\mathrm{i}}\right)$ \\
\hline$-r_{2} x_{1}+x_{7}=0$ & $\left\{x_{7} / 1\right\}$ & 1 \\
$x_{2}-1.1 x_{5}=0$ & $\left\{x_{2} / 1, x_{5} / 1\right\}$ & 2 \\
$-r_{3} x_{3}+x_{6}=0$ & $\left\{x_{6} / 1\right\}$ & 1 \\
$x_{1}-x_{4}-x_{7}+r_{1} x_{7}=0$ & $\left\{x_{1} / 2, x_{4} / 1\right\}$ & 3 \\
$x_{1} x_{2} x_{3}-x_{4} x_{4} x_{6}=0$ & $\emptyset$ & \\
\hline
\end{tabular}

After this symbolic reduction, a step of generation and addition of new constraints is applied to the previous constraint networks.

\section{Generation and Addition of Redundant Constraints}

The addition of redundant constraints to initial $C N$ is often considered to improve the prune of the domains of the possible values for some variables in NCSP. We used it in the polynomial constraints of the models.

In constraint programming languages as CLP(BNR) (Older and Benhamou 1993), NEWTON (Van Hentenryck, Michel, and Deville 1997) and C ++ class library ILOG SOLVER (Ilog 1999), the solving process is based on the adequate combination of local applications of narrowing operators and the propagation of the reduced domains to all the constraints until reaching a stable state. In continuous domains, enumeration technique are used to separate different solutions.

The local application of narrowing operators can improve the computational efficiency when redundant constraints are added. These additions may be performed by hand or in an automated way. An automatic generation of redundancies for polynomial constraints is based on Gröbner basis (Buchberger 1985).

The basic idea of Gröbner basis is to transform a set of polynomials into a certain standard form. Given a system of multivariate polynomial equations, its Gröbner basis is an equivalent system. It is a system that has the same solutions with the same multiplicities. The Gröbner basis are computed by Buchberger's algorithm, that is an algorithm that generalizes both Gaussian elimination for linear multivariate equations and the Euclidean algorithm for univariate polynomial equations. The use of Gröbner basis has the following advantages:

- A Gröbner basis has better computational properties than the original system. In particular, the easy determination whether the system is solvable.

- In over-constrained problems with redundant equations, a Gröbner basis eliminates the redundant ones. 
- In over-constrained and inconsistent problems, the constraint $1=0$ is obtained. It is obviously inconsistent.

- In under-constrained problems, the new problem gives useful information in order to solve the problem.

The principal drawback is the complexity of the algorithm. The computation Gröbner basis for relevant parts of the problem (Benhamou and Granvilliers 1996) and partial Gröbner basis (Benhamou and Granvilliers 1997) have shown computational improvement on some benchmarks.

In this framework, we propose to add these redundant constraints when the performance of the algorithm leads to realistic execution times.

\section{Application of generation and addition of constraint to equilibrium of a weak acid problem}

The application of this Symbolic Processing step allows us to dispose of the identification of consistency of the constraints and also it finds new constraints that are added to the previous one. In this example, the following constraints are added:

$$
\begin{aligned}
-\left[H^{+}\right]+10^{14}\left[H^{+}\right]^{3}-K_{a}-10^{14} c\left[H^{+}\right] K_{a}+10^{14} K_{a}\left[H^{+}\right]^{2} & =0 \\
1-10^{14}\left[H^{+}\right]^{2}+10^{14} c K_{a}-10^{14}\left[H^{+}\right] K_{a}+10^{14} K_{a}\left[O H^{-}\right] & =0
\end{aligned}
$$

\section{Factored Constraints}

In the analysis of engineering systems there are models where we have "factored constraints." By "factored,", we mean that an expression is written in terms that can be multiplied, such as $U_{i} \equiv\left(\Pi_{k} f_{k}=0\right)$. These constraints are rewritten as $U_{i} \equiv O R_{k}\left(f_{k}=0\right)$. That is, each constraint $U_{i}$ is rewritten by a tree compound by $k$ constraints with the operator or in the root. It gives a better domain reduction of the variables for these models.

For example, in the model of countercurrent heat-exchanger the constraint obtained in the symbolic reduction step was

$$
C \equiv\left\{\left(1.1-1 r_{3}+r_{2} r_{3}-r_{1} r_{2} r_{3}\right) x_{1} x_{3} x_{5}=0\right\}
$$

then, the proposed methodology generates a new constraint network of model

$$
x_{1}=0 ; \quad x_{3}=0 ; \quad x_{5}=0 ; \quad 1.1-1 r_{3}+r_{2} r_{3}-r_{1} r_{2} r_{3}=0
$$

It is the disjunction of four constraints. The later numerical treatment only will consider the constraints where we have the queries. In this case the last constraint is only considered. 


\section{Constraint Solving}

In the numerical processing of the constraint network with interval domains, the application of classical interval arithmetic (Moore 1966) can only provide an estimation of the range of the expressions, that is, it only guarantees the solution to be included in the resulting interval. Also, in some cases the range of values of a function is not an interval but a union of intervals. In this work, we use a set of Narrowing Operators and a Constraint Solver Algorithm in order to reduce the widening effect.

\section{Narrowing Operators}

In previous works, some different approximations of arc-consistency have been proposed to obtain better solutions in constraint solving. It states a simple local condition on a constraint and the set of possible values of its variables. A first approximation was used on simple constraints and complex constraints were decomposed in terms of these simple constraints (Hyvönen 1992). The improvement in efficiency and the correctness have been the main goals for others approximations of arc-consistency (Hong 1992; Lhomme 1993; Benhamou and Granvilliers 1996; Van Hentenryck, Michel, and Deville 1997).

In our work the numerical processing is applied to the constraint network $C N_{1}$ obtained by Symbolic Processing step. This processing is based on the definition of Interval Extension (Moore 1966), and Narrowing Operators (Van Hentenryck, Michel, and Deville 1997). The constraints are rewritten as a Natural Interval Extension. It replaces each relational operation by its corresponding interval extension, each real operation by its corresponding interval extension, each variable by an interval variable and each real number by an interval. The interval extension of the relational operations $e_{1} \leq e_{2}$ and $e_{1}=e_{2}$ represents the possibility to be equal and less or equals than respectively. In these interval extensions, we use Narrowing Operators based on box-consistency (Van Hentenryck, Michel, and Deville 1997). It is an approximation of arc consistency that offers a good trade-off between efficiency and pruning.

\section{The Constraint Solver Algorithm}

The previous Narrowing Operators are applied until a fixpoint is reached and the correctness follows from the fact that they do not remove any solutions. The results of the application of the previous Narrowing Operators are propagated to the constraint network until no pruning takes place. But as some solutions may have spurious values, then nondeterministic choices are necessary to enhance the constraint solver. Therefore the key idea is to use a branch and prune algorithm. 
In the proposed methodology, we consider that this algorithm depends on two parameters $\epsilon$ and variable ordering heuristic. $\epsilon$ determines the size of small solutions of the problem. If the operation prune obtains a result that is considered small, then it is a possible solution.

The operation Branch use the parameter variable ordering heuristic to split the next variable. The decisions in this ordering significantly affect the efficiency of the search strategy. Heuristics can be grouped into two categories:

- Static Heuristic, that establishes an ordering before the search starts and maintains this ordering throughout all the search.

- Dynamic Heuristic, that makes selections dynamically during search, the decisions about variable and value orderings are established at each search node.

A well-known static heuristic considers the most constrained variables in the first place, because they are likely more difficult to assign. Inconsistencies are expected to be found at early levels of a tree of potential solutions, where recovering from mistakes is less costly. This has been often used in the bibliography.

It is strongly believed that dynamic variable orderings are more effective than static ones. The most used variable ordering heuristic selects the variable with the minimum number of values in its current domain. However the best variable ordering depends very much on the nature of the problem.

In a previous work about numeric constraint satisfaction problem is proposed (Hyvönen 1992) to select a cutset variable by some criterion (e.g. select the variable with the largest width and split it exhaustively into intervals by some criterion). Another work (Van Hentenryck, Michel, and Deville 1997) used a round-robin heuristic to split the domains of the variables. Our framework allows the election of different splitting strategies by means of the parameter variable splitting ordering. The user can choose static orderings or dynamic orderings, and if the user says nothing then all the variables of the constraint network will be split and the ordering will be based on considering first the most constrained variables.

\section{Experimental Results}

We present here the application of our methodology to simple problems where reasoning steps can be easily tracked and verified. But, the proposed framework is most useful when analysis is performed in complex engineering systems.

The first example involves analysis of an oscillating device. The following ones show the obtained results in the previous semiqualitative models. 


\section{An Oscillating Device Model}

- Description of the system:

The experiments may be performed by a torsion-pendulum, a flying wheel fixed to a certain angular position by a spiral spring, where the deviation from the linear function between angular position and restoring torque is obtained by adding an unbalanced weight. This system, in the case of strong deviations, leads to a point of unstable equilibrium and two stable equilibria in phase-space (Luchner 1987). Figure 6 shows this oscillating device.

It is easy to quantify all torques acting: The spring exerts a torque $M_{1}=$ $-k \phi$ (Hooke's law), and the unbalanced weight exerts an additional torque $\left.M_{2}=\operatorname{mgr} \sin (\phi) \approx \operatorname{mgr}\left(\phi-\phi^{3} / 6\right)\right)$. In this example, the sum of the torques is $M=-k \phi+\operatorname{mgr}\left(\phi-\phi^{3} / 6\right)$. In the equilibrium $M=0$ and the constraint $(-k+m g r) \phi-m g r / 6 \phi^{3}=0$ is held.

- An instance of semiqualitative model:

In a semiqualitative model we may know the following parameters: $g$ is $9.81 \mathrm{~m} / \mathrm{s}^{2}, r$ is $2 \mathrm{~m}, k$ is small and $m$ is large. The representation of this model in the modeling language is:

$$
M_{0} \equiv\left\{\begin{aligned}
\mathrm{C} \equiv & \left\{-k \phi+2 m 9.81\left(\phi-\phi^{3} / 6\right)=0\right. \\
& \operatorname{small}(k), \operatorname{large}(m)\} \\
\mathrm{V} \equiv\{k, \phi, m\} & \\
\mathrm{Q} \equiv & \{\phi ?\}
\end{aligned}\right.
$$

In this problem, according to the experts the intervals corresponding to the qualitative concepts are: $I_{\text {small }}=[0.1,0.2]$ and $I_{\text {large }}=[0.6,1]$. We would like to know the qualitative values of $\phi$ in equilibrium regions. The semiqualitative model can be then:

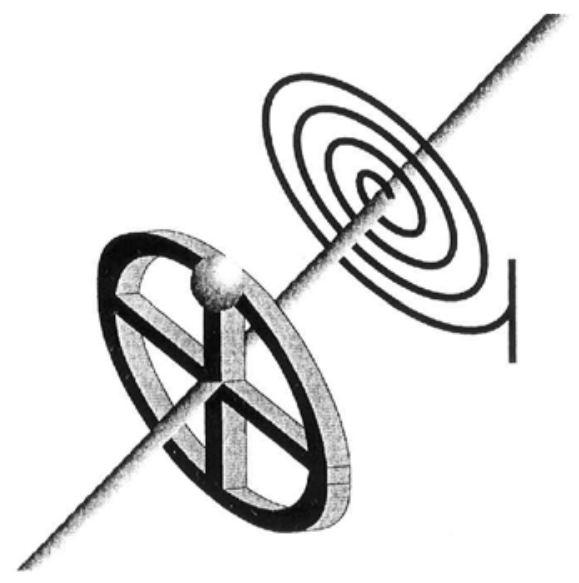

FIGURE 6. Torsion pendulum with unbalanced weight. 


$$
M_{1} \equiv\left\{\begin{aligned}
C= & \left\{-r_{1} r_{3}+2 r_{2} 9.81\left(r_{3}-r_{3}^{3} / 6\right)=0,\right. \\
& k-r_{1}=0, m-r_{2}=0, \phi-r_{3}=0 \\
& \left.r_{1} \in I_{\text {small }}, r_{2} \in I_{\text {large }}\right\} \\
V= & \{Z=\{\}, \\
& \left.Y=\left\{r_{1}, r_{2}\right\}, X=\left\{r_{3}\right\}\right\} \\
Q= & r_{3} ?
\end{aligned}\right.
$$

- Application of constraint solving In this model, the user's query is $r_{3}$ ?. The application of our methodology obtains three equilibrium regions, represented by the real intervals:

$$
I_{1}=[-2.44,-2.42] \quad I_{2}=[-0.000087,0.000088] \quad I_{3}=[2.42,2.44]
$$

The graph of $M(\phi)$ for this example is shown in Figure 7. This function is sandwiched exactly between two univariate real functions defined by the bounds of the parameters in an adequate way. The obtained solutions are represented by means of black boxes. They are very closed to the real solutions.

\section{Weak Acid Model}

In this model, we applied the Symbolic Processing step and obtained a new constraint network $C N_{l}$. Now, we apply to it the Constraint Solver Algorithm. It allows us obtain the interval answers to the query $\left[\mathrm{H}^{+}\right]$?. The result is [0.00125, 0.0174335]. It is complete and its width is within the desired accuracy in the semiqualitative reasoning.

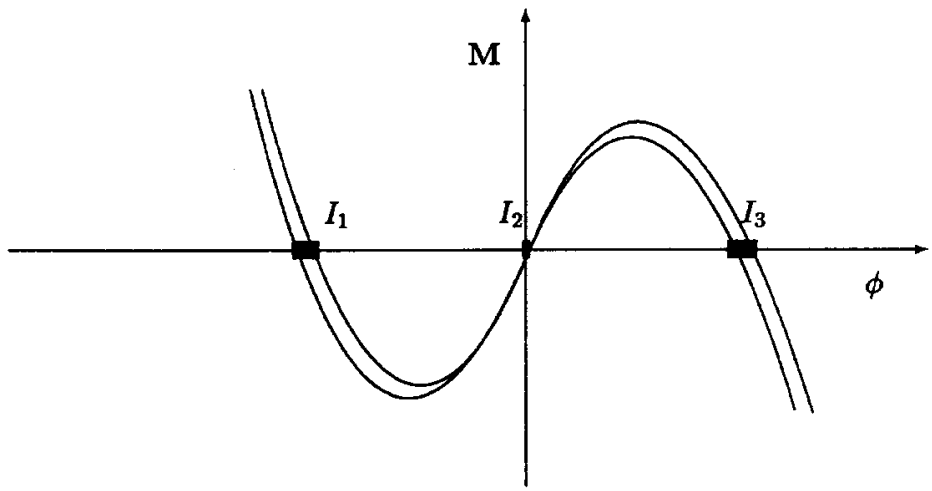

FIGURE 7. Equilibrium regions for an example of oscillating device. 


\section{Countercurrent heat-exchanger Model}

The application of Symbolic Processing step generates the model $M_{2}$. The network constraint obtained to factored constraint was:

$$
x_{1}=0 \quad \text { or } \quad x_{3}=0 \quad \text { or } \quad x_{5}=0 \quad \text { or } \quad 1.1-r_{3}+r_{2} r_{3}-r_{1} r_{2} r_{3}=0
$$

When we apply Constraint Solving step the result is $r_{3} \in[1.089,1.11647]$. This result is complete and we obtain the qualitative information about the relative order of magnitude relation $r_{3}$.

\section{Qualitative Interpretation of the Results}

The described methodology allows to obtain interval solutions for the set of proposed queries. The method of qualitative interpretation of these results consists on finding the qualitative operator or set of qualitative operators of the previously defined ones that minimally cover each of the resulting intervals. The assignment from the qualitative labels to the different intervals is carried out using previously developed methods (Riquelme 1996). The obtained results of the models presented in this work are shown in Table 3. These semiqualitative answers give a significant information when the qualitative and quantitative knowledge is considered.

\section{CONCLUSIONS}

This novel framework may be suitable to capture and to integrate the qualitative and quantitative knowledge of many engineering applications. The wide set of qualitative concepts of the modeling language permits to express this type of knowledge easily. The users' queries allow to derive new knowledge by means of the proposed framework.

The results of the different applications show that the solutions that we have obtained are less ambiguous than the ones obtained using other qualitative techniques. It is mainly due to the symbolic step of our methodology and the application of Narrowing Operators. An adequate election of the heuristic to split variables can improve the efficiency of the numerical

TABLE 3 Table of Results of the Queries of the Semiqualitative Models

\begin{tabular}{lc}
\hline Model & Semiqualitative Answers \\
\hline Weak acid & {$\left[\mathrm{H}^{+}\right]$is medium } \\
Heat-exchanger & $\mathrm{FC}$ is slightly greater than FH \\
Oscillating device & $\phi$ is Negative-Great or Zero or \\
& Positive-Great \\
\hline
\end{tabular}


algorithms. Our reasoning framework permits the explicit description of an variable ordering heuristic.

In the future, we would like to enrich this language with purely qualitative functions and to apply our techniques for semiqualitative analysis of dynamic systems where it is possible to obtain the stability and bifurcations regions. Another possible field of applications of our methodology is the semiqualitative simulation of dynamic systems which must hold a set of constraints.

\section{REFERENCES}

Agell, N. 1998. Estructures matematiques per al model qualitatiu d'ordres de magnitud absoluts. Ph. D. Diss., Universitat Politecnica de Catalunya, Spain.

Aracil, J., and M. Toro. 1993. Métodos cualitativos en dinámica de sistemas. Secretariado de Publicaciones, University of Seville, Spain.

Armengol, J. 2000. Application of modal interval analysis to the simulation of the behaviour of dynamic systems with uncertain parameters. Ph. D. Diss., Girona University.

Benhamou, F., D. McAllester, and P. Van Hentenryck. 1994. CLP intervals revisited. Proceedings of the International Logic Programming Symposium, Ithaca, NY.

Benhamou, F., and L. Granvilliers. 1996. Combining local consistency, symbolic rewriting and interval methods. Proceedings of the AISMC-3, Steyr, Austria, LNCS 1138:44-159, Springer-Verlag.

Benhamou, F., and L. Granvilliers. 1997. Automatic generation of numerical redundancies for non-linear constraint solving. Reliable Computing 3:335-344.

Benhamou, F., and W. Older. 1997. Applying interval arithmetic to real, integer and Boolean constraints. The Journal of Logic Programming 32:1-24.

Berleant, D., and B. J. Kuipers. 1997. Qualitative and quantitative simulation: bridging the gap. Artificial Intelligence 95:215-255.

Biswas, G., R. Kapadia, and W. Yu. 1999. Combined qualitative-quantitative steady state diagnosis of continuous-value systems. IEEE Transactions on Systems, Man and Cybernetics XX:100-118.

Bousson, K. 1993. Raisonnement causal pour la supervision de processus basee sur des modeles. Ph. D. Diss., LAAS-CNRS Toulouse.

Bozzo, L. M., A. Barbat, and L. Torres. 1998. Application of qualitative reasoning in engineering. Applied Artificial Intelligence 12:29-48.

Buchberger, B. 1985. Gröbner bases: An algorithmic method in polynomial ideal theory. In Recent Trends in Multidimensional Systems Theory, 184-232. New York: Reidel.

Collavizza, H., F. Delobel, and M. Rueher. 1999. Comparing partial consistencies. Reliable Computing 5: 213-218.

Dague, P. 1993a. Symbolic reasoning with relative orders of magnitude. Proceedings of the Thirteenth IJCAI, Chambery, France, 1509-1541.

Dague, P. 1993b. Numeric reasoning with relative orders of magnitude. 11th National Conference on Artificial Intelligence, Washington, DC, 541-547.

Dague, P. 1995. Qualitative reasoning: A survery of techniques and applications. AI Communications 8(3/4): 19-192.

Davis, E. 1987. Constraint propagation with interval labels. Artificial Intelligence 32:281-331.

De Kleer, J., and J. S. Brown. 1984. Qualitative physics based on confluences. Artificial Intelligence 24(1-3):7-83.

Dollinger, R., and I. Letia. 1998. Using Tolerance Calculus for reasoning in relative order of magnitude models. Lecture Notes in Artificial Intelligence 1415:399-407.

Dubois, D., and H. Prade. 1989. Order-of-magnitude reasoning with fuzzy relations. Revue d'Intelligence Artificielle (3):69-94.

Forbus, K. D. 1984. Qualitative process theory. Artificial Intelligence 24:85-168.

Freuder, E. C., and A. Mackworth. 1994. Constraint-Based Reasoning. Cambridge, MA: MIT/Elsevier. 
Gasca, R. M., M. Toro, and J. A. Ortega. 1996. Propagacion de restricciones integran conocimiento cualitativo y cuantitativo. Boletín de la AEPIA (6):23-30.

Gasca, R. M. 1998. Razonamiento y simulación en sistemas que integran conocimiento cualitativo y cuantitativo. Ph. D. Diss., University of Sevilla.

Hong, H., and V. Stahl. 1994. Safe starting regions by fixed points and tightening. Computing 53:323-335.

Hyvönen, E. 1992. Constraint reasoning based on interval artithmetic: the tolerance propogation. Artificial Intelligence 58:1-112.

ILOG. 1999. ILOG Solver 4.4 User's Manual. France.

Jussien, N., and O. Lhomme. 1998. Dynamic domain splitting for numeric CSPs. Proceedings of the ECAI98:224-228. Brighton, England.

Kuipers, B. J. 1986. Qualitative Simulation. Artificial Intelligence 29:289-338.

Kuipers, B. J. 1994. Qualitative Reasoning. Modeling and Simulation with Incomplete Knowledge. Cambridge, MA: MIT Press.

Lee, M. H. 2000. Qualitative modelling of linear networks in engineering applications. Proceedings of the ECAI2000, Berlin, Germany, 161-165.

Lhomme, O. 1993. Consistency techniques for numeric CSPs. Proceedings of the 13th IJCAI'93, Chambery, France, 232-238.

Lhomme, O. 1994. Contribution a la resolution de constraintes sur les reels par propagation d'intervalles. Ph. D. Diss., Nice-Sophia University, Antipolis.

Luchner, K. 1987. Introductory experiments on chaotic motion. Proceedings of the Conference on Synergetics Order and Chaos, Madrid, Spain, 41-54.

Moreno, J. 1993. Técnicas cualitativas de supervision: aplicacion a motores marinos. Ph. D. Diss., University of Sevilla.

Marti, P., and M. Rueher. 1997. Concurrent cooperating solvers over reals. Reliable Computing 3:325-333.

Mavrovouniotis, M. L., and G. Stephanopoulos. 1990. Formal order-of-magnitude reasoning in process engineering. In Readings in Qualitative Reasoning about Physical Systems, 323-336. San Mateo, CA: Morgan Kaufmann.

Missier, A., N. Piera, and L. Trave. 1989. Order of magnitude algerbras: A survey. Revue d'Intelligence Artificielle 3(4):95-109.

Moore, R. E. 1966. Interval Analysis. Series in Automatic Computation. Englewood Cliffs, NJ: PrenticeHall.

Older, W. J., and F. Benhamou. 1993. Programming in CLP(BNR). Proceedings of P PCP'93 Newport, RI.

Ortega, J. A. 2000. Patrones de comportamiento temporal en sistemas dinBmicos con restricciones. Ph. D. Diss., University of Sevilla.

Piera, N., M. Sánchez, and L. Travé-Massuyès. 1991. Qualitative operators for order of magnitude calculers: Robustness and precision. Proceedings of the 43rd IMACS World Congress on Computation and Applied Mathematics, Dublin, Ireland, 1-6.

Piera, N. (ed.) 1995. Current Trends in Qualitative Reasoning and Applications. Nuria Piera, Spain.

Raiman, O. 1986. Order of magnitude reasoning. Proceedings of the 5th National Conference on Artificial Intelligence, Philadelphia, PA, 100-104.

Raiman, O. 1991. Order of magnitude reasoning. Artificial Intelligence 51:11-38.

Ratschek, H., and J. Rokne. 1984. Computer Methods for the Range of Functions. England: Ellis Horwood.

Riquelme, J. C. 1996. Obtención de información cualitativa a partir de datos cuantitativos: Aplicación al análisis de sistemas complejos. Ph. D. Diss., University of Sevilla.

Sánchez, M., F. Prats, and N. Piera. 1996a. Mixing the Absolute and Relative Order of Magnitude Reasoning in Qualitative Models. Research Report MA2-IT-96-0005, UPC, Barcelona.

Sánchez, M., F. Prats, and N. Piera. 1996b. Degrees of negligibility in qualitative models. Proc. Jornads Hispano Francesas, Barcelona, Spain.

Sánchez M., F. Prats, and N. Piera 1998. Negligibility relations between numbers and qualitative labels. Lecture Notes in Artificial Intelligence 1415:367-376.

Struss, P. 1989. Structuring of models and reasoning about quantities in qualitative physics. Ph.D. Diss., University of Kaiserlautern.

Struss, P. 1990. Problems in interval-based qualitative reasoning. Qualitative Reasoning about Physics Systems. San Mateo, CA: Morgan Kaufmann. 
Travé-Massuyès, L., and N. Piera. 1989. The order of magnitude models as qualitative algebras. Proceedings of the 11th Joint Conference on Artificial Intelligence, Detroit, MI, 1261-1266.

Travé-Massuyès, L., P. Dague, and F. Guerrin. 1997. Le raisonnement qualiftatif pour les sciences de l'ingenieur. Paris: Hermes.

Van Hentenryck, P., and L. Michel. 1995. Newton: Constraint Programming over Nonlinear Real Constraints. Technical Report No. CS-95-25, Brown University.

Van Hentenryck, P., D. McAllester, and D. Kapur. 1995. Solving Polynomial Systems using a Branch and Prune approach. Technical Report No. CS-95-01, Brown University.

Van Hentenryck, P., L. Michel, and Y. Denville. 1997. Numerica. A Modeling Language for Global Optimization. Cambridge, MA: MIT Press.

Williams, B. C. 1991. A theory of interactions: Unifying qualitative and quantitative algebraic reasoning. Artificial Intelligence 51:39-94. 